\title{
Model of the Aerodynamic Behavior of a Pararotor
}

\author{
Vicente Nadal-Mora / Ángel Sanz-Andrés / Álvaro Cuerva ${ }^{\ddagger}$
}

\begin{abstract}
A simple semi-empirical model for the aerodynamic behavior of a low-aspect ratio pararotor in autorotation at low Reynolds numbers is presented. The paper is split into three sections: Sec. II deals with the theoretical model derivation, Sec. III deals with the wind-tunnel measurements needed for tuning the theoretical model, and Sec. IV deals with the tuning between the theoretical model and the experimental data. The study is focused on the effect of both the blade pitch angle and the blade roughness and also on the stream velocity, on the rotation velocity, and on the drag of a model. Flow pattern visualizations have also been performed. The value of the free aerodynamic parameters of the semi-empirical model that produces the best fit with the experimental results agrees with the expected ones for the blades at the test conditions. Finally, the model is able to describe the behavior of a pararotor in autorotation that rotates fixed to a shaft, validated for a range of blade pitch angles. The movement of the device is found to be governed by a reduced set of dimensionless parameters.
\end{abstract}

\section{Nomenclature}

$B$

$C_{D}$

$C_{D 0}$

$C_{\mathrm{DM}}$

$C_{\text {DM max }}$

$C_{L \alpha}$

$c$

D $\quad=$ blade chord

dif $=$ difference between theoretical and experimental values

$F_{T} \quad=$ tangential force

$k=$ velocity ratio

$k_{\text {min }}=$ minimum velocity ratio

$L \quad=$ lift

$N=$ number of cases considered for the matching

$p_{i} \quad=$ parameter $\left(k, \bar{V}_{v},{\overline{U_{T}}}^{2}\right)$

$p_{i \exp }\left(\beta_{j}\right)=$ experimental value of the parameter $p_{i}$ for the blade pitch angle $\beta_{j}$

$p_{i \text { the }}\left(\beta_{j}\right)=$ theoretical value of the parameter $p_{i}$ for the blade pitch angle $\beta_{j}$

$R e_{P} \quad=$ Reynolds number

$r \quad=$ radius of the blade reference section

$S_{P} \quad=$ area of the two blades
$R \quad=$ model radius

$S_{D} \quad=$ actuator disk area

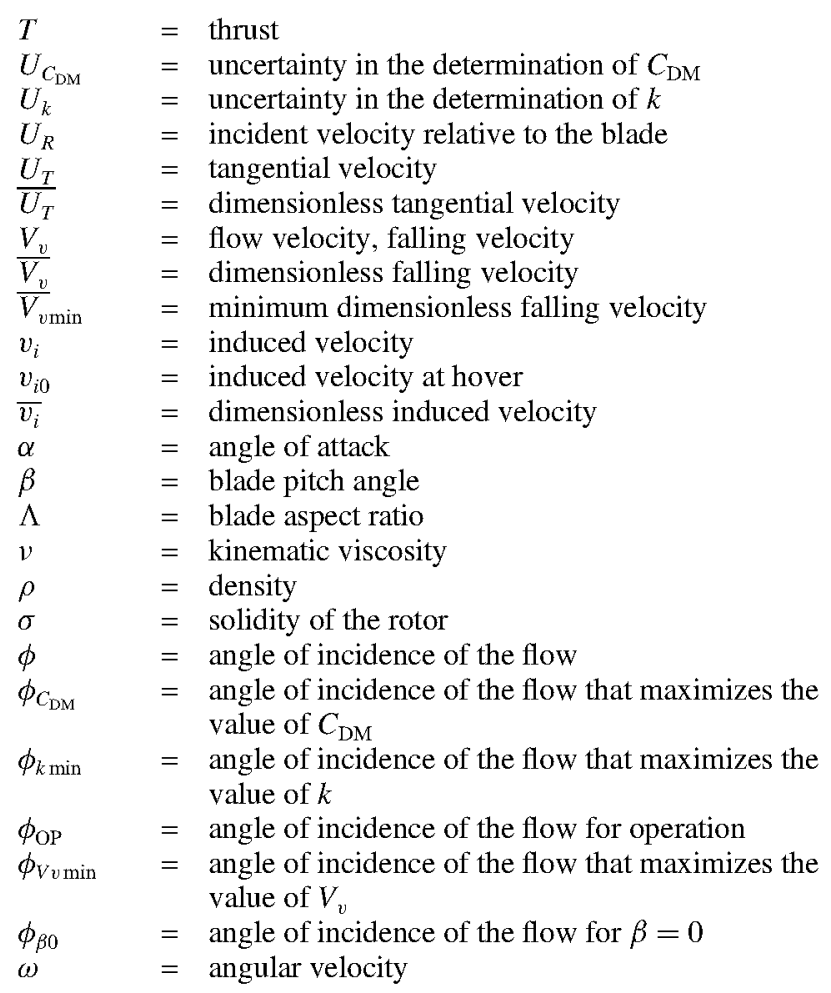

\section{Introduction}

Received 28 November 2005; revision received 3 May 2006; accepted for publication 5 June 2006. Copyright $(\mathcal{C} 2006$ by the American Institute of Aeronautics and Astronautics, Inc. All rights reserved. Copies of this paper may be made for personal or internal use, on condition that the copier pay the $\$ 10.00$ per-copy fee to the Copyright Clearance Center, Inc., 222 Rosewood Drive, Danvers, MA 01923; include the code $\$ 10.00$ in correspondence with the CCC.

*Professor, Aeronautical Engineering, Área Departamental Aeronáutica, Facultad de Ingeniería, Calle 116 entre 47 y 48 . Member AIAA.

${ }^{\dagger}$ Professor, Aeronautical Engineering, Instituto Universitario de Microgravedad "Ignacio Da Riva," Escuela Técnica Superior de Ingenieros Aeronáuticos. Member ALAA.

${ }^{\ddagger}$ Professor, Aeronautical Engineering, Instituto Universitario de Microgravedad "Ignacio Da Riva," Escuela Técnica Superior de Ingenieros Aeronáuticos.

\begin{abstract}
A pararotor is a device like an unpowered helicopter rotor that spins in an autorotation regime. A drag force in the direction of the incident flow is generated over the rotor. The drag exerted over the rotor is greater if it spins than if it does not. Therefore the rotational motion is effective to slow down falling bodies in the atmosphere and also to stabilize their trajectory. To achieve both effects in a proper way, it is necessary to study the characteristics of the device in motion, to find which parameters affect its performances and to quantify the influence of each parameter.

The intended application of this pararotor is to measure winds, emissions, and atmospheric parameters for air traffic support, although the principle could be used for other applications, such as the recovery of reentry vehicles or guidance and control of
\end{abstract}


projectiles. Among these last applications, there are the studies carried out by Crimi [1], who studied a rotating body with only one wing for submunition applications, and Karlsen et al. [2], who also worked on winged bodies for submunition applications. They talked about the advantages of the pararotor over the parachute: lower sensitivity to lateral winds, parachute deployment problems, lower precession movements, and higher falling velocity.

Among the reentry applications there are many studies related to the use of rotating parachutes, like the one presented by Pepper Jr. [3]. Rotating parachutes for decelerating and controlling falling bodies were also studied by Shpund and Levin [4-7].

In addition to the mentioned works, it has not been possible to find in the literature experimental studies related to pararotors or autorotating-winged devices with characteristics similar to the one presented here, that is, rotors with low aspect ratio blades. However, there are works related to large aspect ratio bodies like the samara wings (Rosen and Seter [8,9]). The samaras are a solution adopted by some plants to transport and spread their seeds, which are carried inside "wings" that fly in the autorotation regime.

Analyzing the mentioned devices, the blades of the pararotors usually have a much lower aspect ratio than the samaras and the helicopter rotors. Furthermore, the samaras work at lower Reynolds numbers and at larger angles of attack than the device studied here, and helicopter rotors used to work at a larger Reynolds number than pararotors. In addition to that, the blade element theory developed for long span rotating wings, like helicopter rotor, is not applicable to the pararotor problem considered in this paper because of the low aspect ratio of pararotor blades. For that reason, a different approach has been followed here, which has, however, some similarities with the blade element theory.

As mentioned, an investigation about the behavior of low aspect ratio rotating-winged devices is presented in this paper. There are at least two reasons that justify following this line of investigation: 1) there is not enough published research concerning this subject and 2) the possible applications of the device.

The research has been carried out (and validated) for a range of blade pitch angles between 2 and $8 \mathrm{deg}$, which is the assumed range of application of the device. Increasing the blade pitch angle further produces an excessively large rotation velocity and a poor equivalent pararotor drag coefficient; hence, a poor deceleration rate is achieved.

A simple theoretical model based on some experimental data, which describes the behavior of a pararotor, is developed in Sec. II. Given the pararotor's geometric and aerodynamic characteristics, such as blade dimensions, blade drag coefficient, blade pitch angle, etc., the model allows us to determine the ratio between the falling velocity and the rotation velocity of the device, both the dimensionless falling and rotation velocities, the pararotor drag coefficient (drag in the falling direction), the blade angle of attack, and the angle of incidence of the flow to the blades. The aerodynamic parameters of the blades could be determined by testing the blades separately from the pararotor body, but as these data are not currently available (and the application to a configuration in rotational motion could cast doubts about the validity of the extrapolation of the data obtained from translational motion tests), it has been necessary to follow a different approach based on the determination of the parameters that better match the results given by the model to the corresponding ones obtained from experimental tests of a pararotor (Sec. IV).

The experimental work, performed in a wind tunnel (Sec. III), consists in the determination of the rotation velocity and the drag for different falling velocities and blade pitch angles of a pararotor that spins fixed to a rotating shaft. Blades with different roughness have been tested to clarify the influence of the parameters concerned.

Finally, the contribution of the work reported in the paper is the development of a simple mathematical model that allows us to predict the performance of a pararotor with different aerodynamic characteristics and, at the same time, gather some knowledge about the behavior of low aspect ratio rotating-winged devices.

It is worth mentioning that the model has been validated for a pararotor with the characteristics of the one used for the tests. It is still to be shown that the model could be used to describe the behavior of a pararotor having different blade planforms, numbers of blades, blade cross sections, larger blade pitch angles, etc.

This paper is structured as follows: in Sec. II, the theoretical model is presented; in Sec. III, the experimental work is summarized; in Sec. IV, the best-fitting parameters are determined; and finally, conclusions on the results of the work are drawn.

\section{Theoretical Model}

The system studied here consists of a rotating body with low aspect ratio wings, called blades, that falls at constant velocity and steady attitude (steady autorotation). The aerodynamic forces generated by the blades make the body to rotate. The model used for the experimental tests is shown in Fig. 1. The numbers in the figure indicate the following: 1 is the cylinder; 2 is the blade; 3 is the balance casing; and 4 is the base of the wind tunnel. The air flows from left to right.

The aerodynamics of low aspect ratio rotating wings is not well known. The flow pattern for these rotors differs widely from large aspect ratio wing rotors, where the blade element theory is valid. This theory assumes the existence of an almost two-dimensional flow in each plane perpendicular to the blade span. In the case of a low aspect ratio rotating wing, flow visualizations show a three-dimensional pattern with flow detached at the leading edge of the blades, as it would be the flow around a delta wing. A pure theoretical model that includes all the details of this flow would be too much complex, and even unattainable, to be developed at a first stage, where just the most relevant characteristics of the phenomenon are investigated. Therefore, just a simple model, based on a semi-empirical approach, has been considered.

The aerodynamic forces acting on the blades have been modeled in a rather simple conceptual way: simple curves relating both the net lift and drag forces over the blade vs the angle of attack have been assumed, whose main parameters are to be determined by correlation with experimental data. The mathematical model has been developed by using elements of the momentum theory to deduce the induced velocity due to the wake and by establishing the equilibrium of the forces that act over the blades required to attain the autorotation state.

The aim of the theoretical model is to determine the performance parameters as a function of the blade pitch angle and the geometric and aerodynamic properties of the configuration.

The main assumptions are as follows:

1) The simple model of the momentum theory (actuator disk), considered in rotor aerodynamics theory, is valid, and therefore

$$
T=-2 \rho S_{D} v_{i}\left(V_{v}+v_{i}\right)
$$

2) The aerodynamic forces (see Fig. 2) acting on the pressure center of the blades are the net lift of the wing

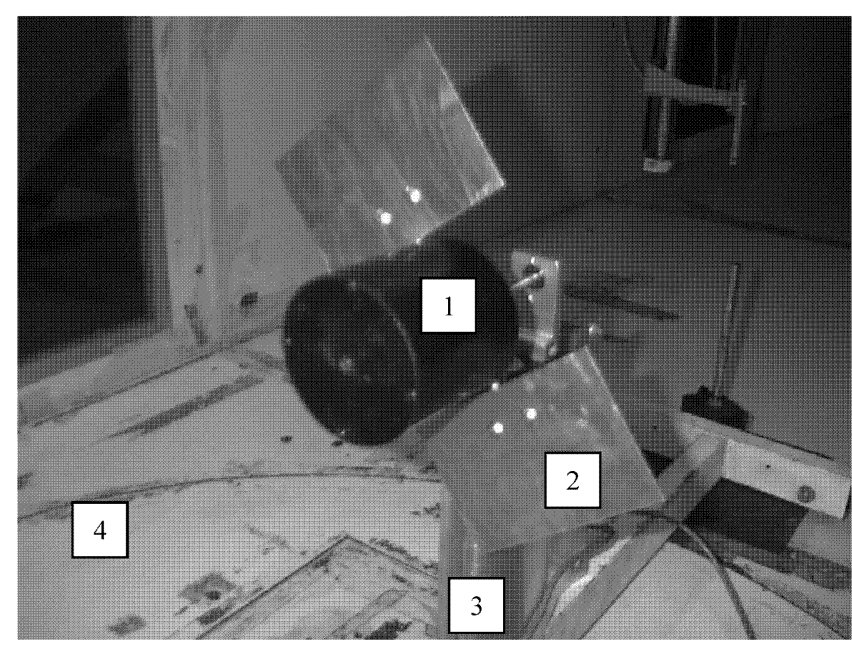

Fig. 1 View of the model mounted inside the wind tunnel. 


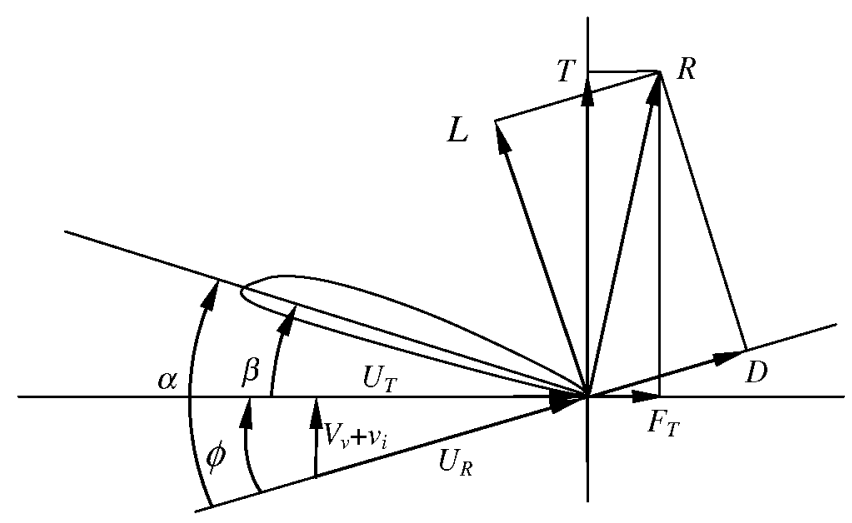

Fig. 2 Angles, net forces, and velocities as seen from the blade.

$$
L=\frac{1}{2} \rho U_{R}^{2} S_{P} C_{L \alpha} \alpha
$$

and the net drag of the wing

$$
D=\frac{1}{2} \rho U_{R}^{2} S_{P} C_{D}
$$

Here the net aerodynamic effect on the whole blade is considered, instead of the forces of each blade section. This assumption is the main difference with the blade element theory, which cannot be applied here because the flow cannot be assumed to be twodimensional, that is, the same pattern in each plane perpendicular to the span. The position of the pressure center is unknown and is to be determined by correlation with the experimental data.

Therefore the net thrust is

$$
T=L \cos \phi+D \sin \phi
$$

and the net tangential force is

$$
F_{T}=-L \sin \phi+D \cos \phi
$$

The angle of incidence of the flow is given by

$$
\tan \phi=-\frac{V_{v}+v_{i}}{\omega r} \approx \phi
$$

The second equality is valid when $\phi \ll 1$. In this case also $U_{R} \approx \omega r=U_{T}$.

In autorotation at a constant falling velocity, the vertical and tangential equilibrium lead to the following relationships:

$$
\begin{gathered}
T \approx L=\frac{1}{2} \rho(\omega r)^{2} S_{P} C_{L \alpha} \alpha \\
T \phi=D
\end{gathered}
$$

where

$$
\alpha=\phi+\beta
$$

The following dimensionless parameters are considered:

$$
\begin{gathered}
k=-\frac{V_{v}}{U_{T}} \\
\overline{V_{v}}=\frac{V_{v}}{v_{i 0}} \\
\overline{U_{T}}=\frac{U_{T}}{v_{i 0}} \\
\overline{v_{i}}=\frac{v_{i}}{v_{i 0}}
\end{gathered}
$$

where

$$
v_{i 0}=\sqrt{\frac{T}{2 \rho S_{D}}}
$$

is the induced velocity at hover. After some manipulation the following set of relationships are obtained for both the input $\beta$ and the performance parameters, as functions of the angle of incidence of the flow:

$$
\begin{gathered}
\beta=\frac{1}{\phi} \frac{C_{D}}{C_{L \alpha}}-\phi \\
{\overline{U_{T}}}^{2}=\phi \frac{1}{(\sigma / 4) C_{D}} \\
\overline{v_{i}}=\phi^{-\frac{3}{2}} \frac{1}{\sqrt{(\sigma / 4) C_{D}}}
\end{gathered}
$$

$$
\begin{aligned}
& k=\phi+\frac{\overline{v_{i}}}{\overline{U_{T}}}=\phi+\frac{\phi^{-\frac{3}{2}} \sqrt{1 /(\sigma / 4) C_{D}}}{\phi\left[1 /(\sigma / 4) C_{D}\right]}=\phi+\frac{1}{\phi^{2}} \frac{\sigma}{4} C_{D} \\
& -\overline{V_{v}}=k \overline{U_{T}}=\left(\phi+\frac{1}{\phi^{2}} \frac{\sigma}{4} C_{D}\right) \sqrt{\phi \frac{1}{(\sigma / 4) C_{D}}}=\frac{\phi^{\frac{3}{2}}}{\sqrt{(\sigma / 4) C_{D}}} \\
& +\sqrt{\frac{\sigma}{4} C_{D}} \phi^{-\frac{3}{2}}
\end{aligned}
$$

where

$$
\sigma=\frac{S_{P}}{S_{D}}
$$

is the solidity of the rotor. In this case the blades do not reach the rotation center because it is occupied by the cylinder that forms the pararotor body. Therefore, the actuator disk area is the area of the circular sector swept by the blades.

The preceding expressions show the influence of the relevant parameters: the solidity, the drag coefficient, and the lift slope.

There are several interesting values of $\phi$ that characterize the shape of the $k(\phi), V_{v}(\phi)$, and $\beta(\phi)$ curves. These are $\phi_{k \min }, \phi_{V v \min }$, which make minimum the values of $k(\phi)$ and $V_{v}(\phi)$, respectively, and $\phi_{\beta 0}$, which is the value of the flow incidence angle when $\beta=0$. The expressions for these values are

$$
\phi_{\beta 0}=\sqrt{\frac{C_{D}}{C_{L \alpha}}}
$$

$$
\phi_{k \min }=\sqrt[3]{\frac{\sigma}{2} C_{D}}
$$

$$
\phi_{V v \min }=\sqrt[3]{\frac{\sigma}{4} C_{D}}
$$

For the pararotor to start to rotate, $\beta$ should be negative; therefore, only the values of $\phi>\phi_{\beta 0}$, leading to $\beta<0$, are of interest. As $C_{D} / C_{L \alpha}$ increases, $\phi_{\beta 0}$ also increases; therefore, for a given $\beta$, the flow incidence angle also increases. It is also shown that $\phi_{V v \text { min }}$ is less than $\phi_{k \text { min }}\left(\phi_{k \text { min }}=2^{1 / 3} \phi_{V v \text { min }}\right)$. And in most of the cases of interest the relationships $\phi_{\beta 0}<\phi_{V v \text { min }}<\phi_{k \text { min }}$ hold.

The minimum values of $k$ and $-\overline{V_{v}}$, from Eqs. (18), (19), (22), and (23), are

$$
k_{\min }=\frac{3}{2}\left(\frac{\sigma}{2} C_{D}\right)^{\frac{1}{3}}
$$




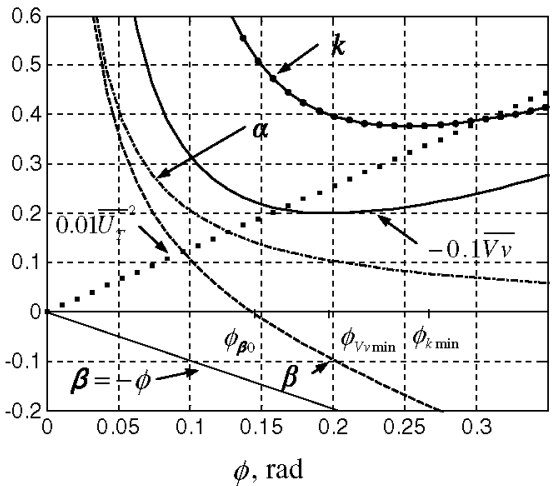

a) $C_{D}=0.07, C_{L \alpha}=3.4$, and $\sigma=0.45$

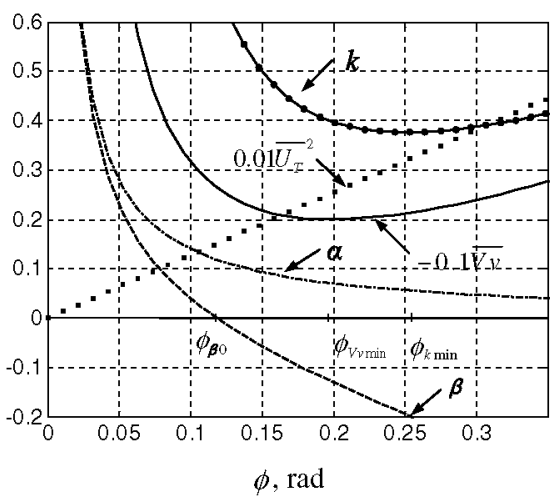

c) $C_{D}=0.07, C_{L \alpha}=5$, and $\sigma=0.45$

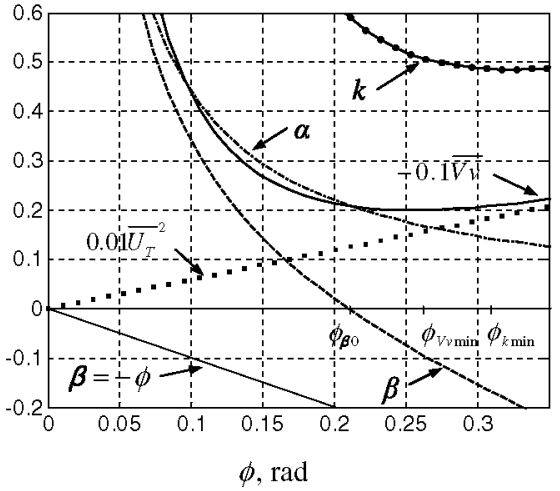

b) $C_{D}=0.15, C_{L \alpha}=3.4$, and $\sigma=0.45$

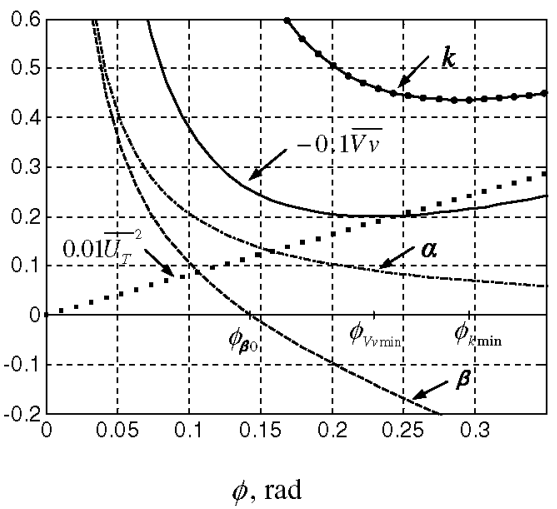

d) $C_{D}=0.07, C_{L \alpha}=3.4$, and $\sigma=0.7$

Fig. 3 Variation of ${\overline{U_{T}}}^{2},-\overline{V_{v}}, \beta, k$, and $\alpha$ vs $\phi$.

$$
-{\overline{V_{v \min }}}=2
$$

$k_{\min }$ is a slow varying function of $C_{D}$ and $\sigma$ due to the $1 / 3$ exponent. Note that $\bar{V}_{v \text { min }}$ is independent of the parameters involved in the problem. The variation of the mentioned performance parameters as a function of $\phi$ is shown in Fig. 3 .

It is observed that when $C_{D}$ increases (compare Figs. 3a and 3b) the slope of the curve ${\overline{U_{T}}}^{2}$ vs $\phi$ decreases (the model rotation velocity decreases) and obviously $k_{\min }$ increases; $\phi_{k \min }, \phi_{V v \min }$, and $\phi_{\beta 0}$ move to the right. For a given value of the blade pitch angle, the angle of attack increases as $C_{D}$ increases. This can lead to nonlinearities in the aerodynamic behavior (stall). When $C_{L \alpha}$ increases (compare Figs. $3 \mathrm{a}$ and $3 \mathrm{c}$ ), the curves of ${\overline{U_{T}}}^{2}, k$, and $-\overline{V_{\nu}}$ vs $\phi$ remain unchanged, while the only change is observed in $\phi_{\beta 0}$, which decreases. Comparing Figs. 3a and 3d; note that $C_{D}$ and $\sigma$ have the same effect on the curves $\overline{U_{T}}, k$, and $-\overline{V_{v}}$ vs $\phi$, whereas the curves $\alpha(\phi)$ and $\beta(\phi)$ are not influenced by $\sigma$. In all cases, as the blade pitch angle increases it approaches the flow incidence angle, and the angle of attack decreases, approaching zero. The physical interpretation is that a small lift (obtained thanks to a small angle of attack) is enough to compensate the drag because the projection of the lift along the blade trajectory, $L \sin \phi$, increases due to a larger value of the flow incidence angle.

The effect of $C_{D}$ and $C_{I \alpha}$, respectively, on the velocity ratio, as a function of the blade pitch angle, is shown in Fig. 4. Observe that almost constant values of $k$ are attained in the range $0.1<-\beta<0.2$, whereas $k$ shows some variations in the range $-\beta<0.1$. Here is also reproduced the previous result concerning the reduction of the rotation rate ( $k$ increases) when the drag increases (Fig. 4a).

In Fig. 5 the relation between the blade angle of attack and the blade pitch angle is shown for different values of the drag coefficient (Fig. 5a) and the slope of the lift curve (Fig. 5b). Observe (Fig. 5a) that the predicted angles of attack are large for large values of $C_{D}$ and for small values of the blade pitch angle. These large values are close to the limit of validity of the linear approximation of the $C_{L}$ vs $\alpha$ relation. The increase of the angles of attack can be traced back to the reduction of the rotation velocity (see Fig. 2).

On the other hand, as shown in Fig. $5 \mathrm{~b}$, the influence of $C_{L \alpha}$ is relatively small in the range considered: $3.4<C_{L \alpha}<5$, which is the range of interest.

The variation with the solidity has not been presented here because it has no influence in the relation between $\alpha$ and $\beta$.

Another performance parameter of interest is the equivalent pararotor drag coefficient that is defined as follows:

$$
C_{\mathrm{DM}}=\frac{T}{(1 / 2) \rho V_{v}^{2} S_{p}}
$$

and therefore

$$
C_{\mathrm{DM}}=\frac{U_{T}^{2}}{V_{v}^{2}} C_{L \alpha} \alpha=\frac{C_{L \alpha} \alpha}{k^{2}}=\frac{C_{D}}{\phi^{3}+\left(1 / \phi^{3}\right)\left[(\sigma / 4) C_{D}\right]^{2}+(\sigma / 2) C_{D}}
$$

The maximum value of $C_{\mathrm{DM}}, C_{\mathrm{DM} \max }=1 / \sigma$, occurs at the angle of incidence of the flow $\phi=\phi_{C_{\mathrm{DM}}}$

$$
\phi_{C_{\mathrm{DM}}}=\left(\frac{\sigma}{4} C_{D}\right)^{1 / 3}=\phi_{V v \min }
$$

Note that the maximum drag coefficient appears at the same angle of incidence of the flow that gives the minimum falling velocity $\phi_{V v \text { min }}$. It should be remarked that, according to this model, $C_{D}$ does not have an influence on the value of $C_{\mathrm{DM} \text { max }}$; however, if $C_{D}$ increases, the maximum value appears at larger values of the angle of incidence of the flow and, as shown in Fig. 5a, large values of $\alpha$, thus approaching blade stall.

These graphs can help to design a pararotor under some given requirements. As an example, consider that the aim is to determine $\beta$ for a minimum value of the falling velocity with a minimum rotation 


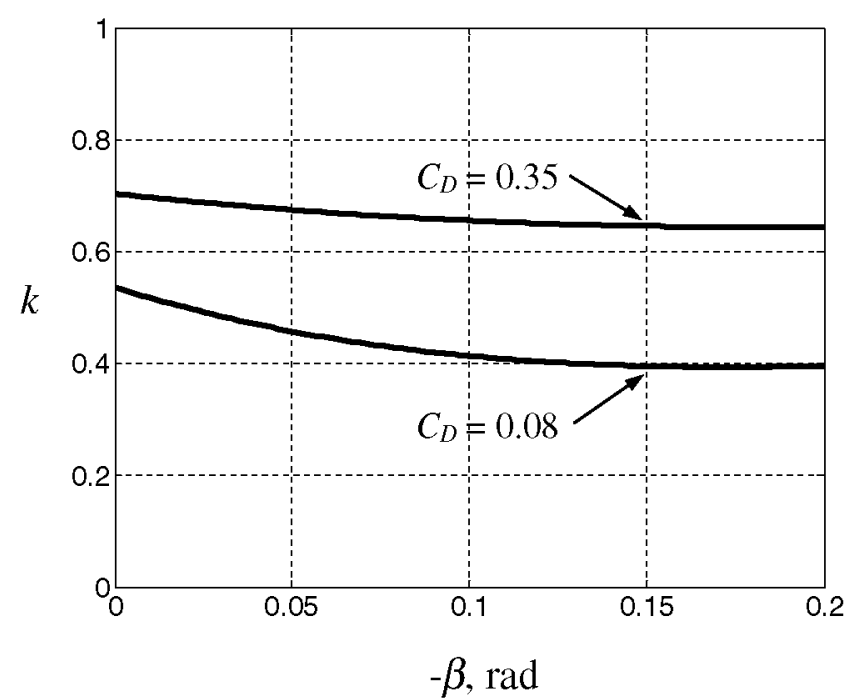

a) $\mathrm{C}_{L \alpha}=3.4$ and $\sigma=0.45$

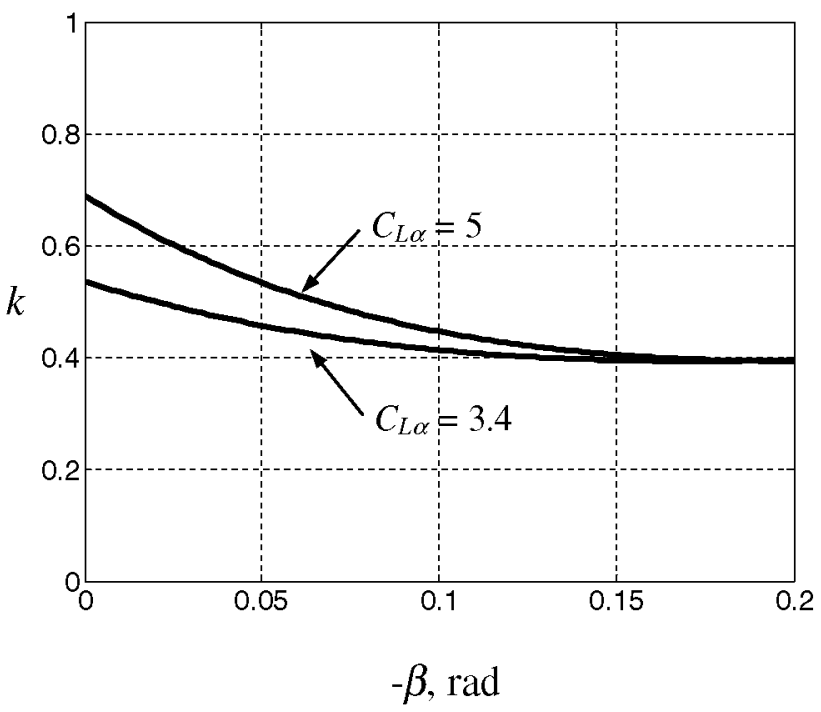

b) $\mathrm{C}_{D}=0.08$ and $\sigma=0.45$

Fig. 4 Variation of the velocity ratio vs the blade pitch angle.

velocity. The minimum value for $-\overline{V_{v}}$ is 2 [Eq. (25)], and it is obtained at $\phi_{v v \min }$, coincident with the maximum value of the equivalent pararotor drag coefficient. On the other hand, to obtain the minimum rotation velocity, $\sigma C_{D}$ should be as large as possible [Eq. (16)] and $k$ should be as large as possible too [Eq. (10)], and so the value of $\phi$ for operation should be as distant as possible from $\phi_{k \text { min }}$. As $\beta$ must be negative for the pararotor to start to rotate, $\beta$ should be as close to zero as possible. In addition to that, and fortunately, $C_{L \alpha}$ is an independent parameter that can help to attain the desired pararotor performance through its influence on the blade pitch angle [Eq. (15)]. In this case, the philosophy used to determine its value is to find the value that places $\phi_{\beta 0}$ closer to $\phi_{V v \min }$ and, therefore, to reduce the blade pitch angle as much as possible.

As it has been shown, the model just presented allows us to obtain simple analytical solutions, which help in understanding the behavior of the system. However, to improve the quantitative results of the model, regarding the comparison with the experimental results, some modifications have been introduced.

1) It is assumed that $C_{D}=C_{D 0}+a \alpha^{2} . C_{D 0}$ and $a$ are two parameters to be determined by matching the model to the experiments.

2) From the flow visualizations it is observed that the blades work more like delta wings than like rectangular ones, but the effective aspect ratio of the blades to be employed is difficult to determine. For

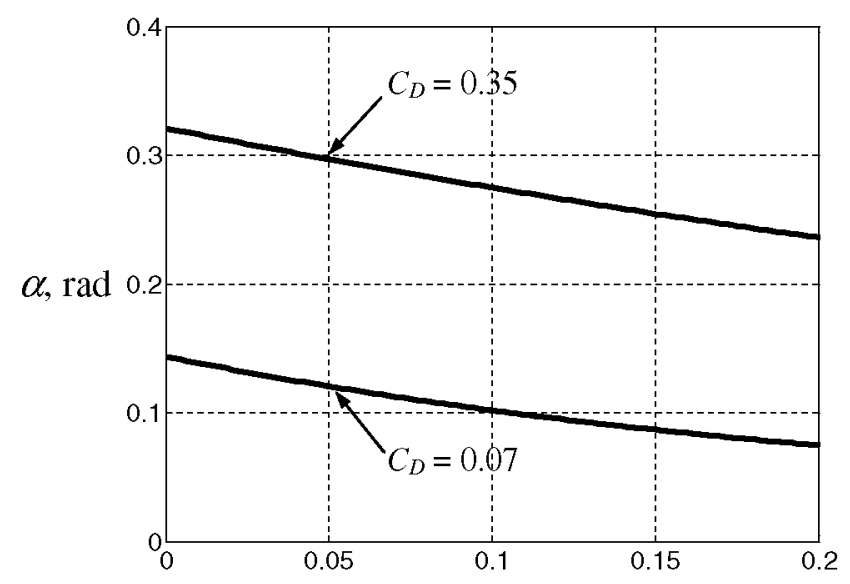

$-\beta, \mathrm{rad}$

a) $\mathrm{C}_{L \alpha}=3.4$ and $\sigma=0.45$

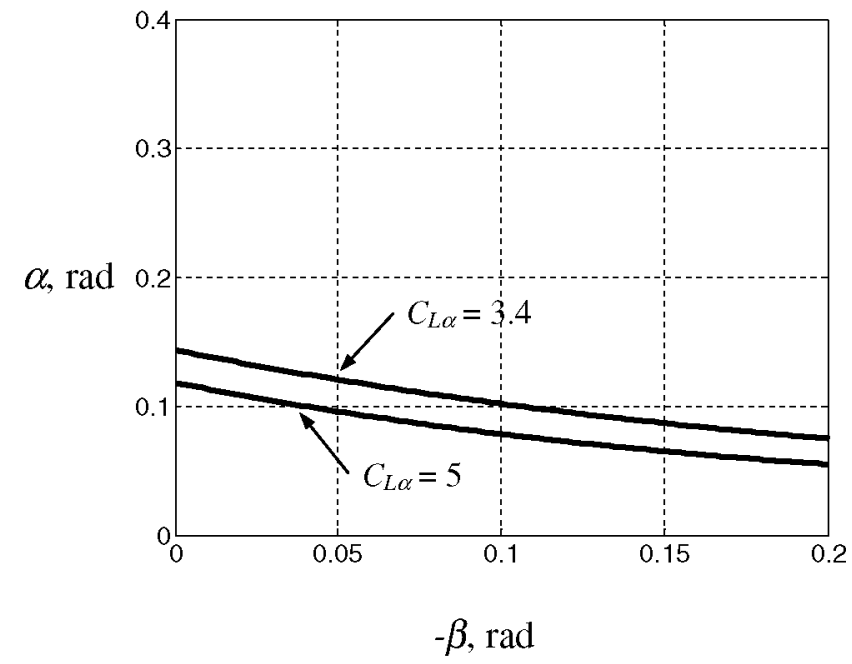

b) $\mathrm{C}_{D}=0.07$ and $\sigma=0.45$

Fig. 5 Variation of the angle of attack vs the blade pitch angle.

this reason, the aspect ratio is not considered as an input parameter of the model.

3) To check the results of the fitting, the expression $C_{L}=$ $(\pi / 2) \Lambda \alpha+4 \alpha^{2} \quad[10]$ has been taken as a reference; then $C_{L \alpha}=(\pi / 2) \Lambda+8 \alpha$. The values of $C_{L \alpha}$ obtained from the fitting between the model and the experiments correspond to reasonable values of $\alpha$ and $\Lambda$.

It is also worth mentioning that the effect of both the lateral skin friction of the cylinder and the friction of the bearings, on which the body is mounted for the tests, has also been assessed and, in consequence, neglected [11].

\section{Experimental Tests and Results}

\section{A. Model Description and Experimental Setup}

The experiments have been performed in the wind tunnel of the Área Departamental Aeronáutica, Facultad de Ingeniería, Universidad Nacional de La Plata, Argentina. It is a $37.3 \mathrm{~kW}$ closedcircuit closed-test-section wind tunnel with a $1.4 \mathrm{~m}$ wide by $1 \mathrm{~m}$ high by $7.2 \mathrm{~m}$ long parallelepipedic test chamber. The wind velocity at the test section can be varied continuously up to $18 \mathrm{~m} / \mathrm{s}$.

The purpose of the tests is to analyze the performance of a model that has a main body, cylindrical in shape, and two variable pitch blades, mounted at the downstream base of the cylinder. The blades are rectangular in shape, of fixed dimensions for all the tests. The model is fixed to a shaft that is mounted on two bearings to a 


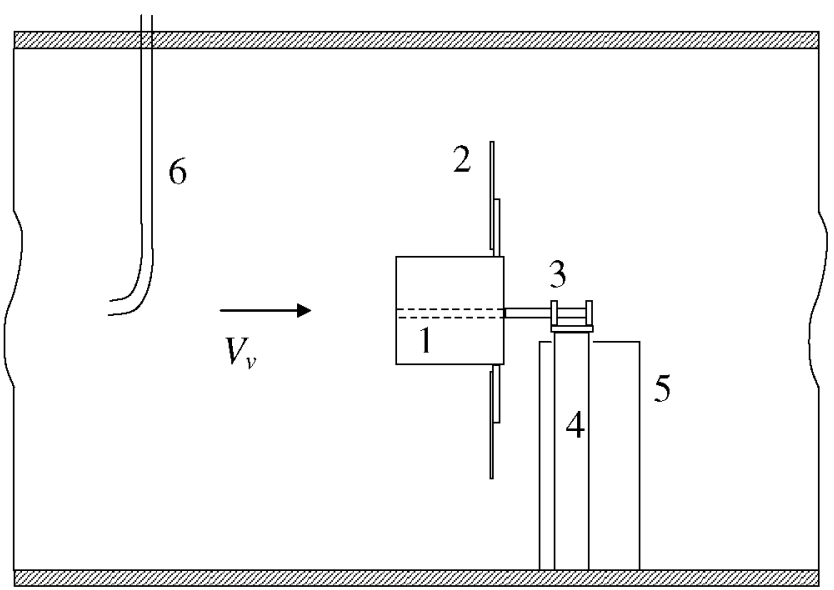

Fig. 6 Sketch of the experimental setup.

structure, which is fixed to a force balance. The model spins freely under the action of the wind. The cylinder has the same height and diameter: $88 \mathrm{~mm}$. The blades have an $88 \mathrm{~mm}$ span, a $138 \mathrm{~mm}$ chord, and a $1 \mathrm{~mm}$ thickness. A sketch of the experimental setup is displayed in Fig. 6. The numbers in the figure indicate the following: 1 is the main body; 2 is the blade; 3 is the rotation shaft; 4 is the balance; 5 is the balance casing; and 6 is the pitot tube.

During the tests, both the drag force exerted over the model and its rotation velocity, together with the flow conditions (flow velocity, temperature, etc.), have been measured.

\section{B. Definitions, Measured Parameters, and Instrumentation}

The following parameters have been measured: the rotation velocity, by a laser tachometer [resolution $0.1 \mathrm{rpm}(<1000 \mathrm{rpm})$, $1 \mathrm{rpm}(>1000 \mathrm{rpm})]$; the blade pitch angle, with a goniometer (resolution $1 \mathrm{deg}$ ); the flow velocity, by a standard NPL pitot-tube type and a micromanometer (resolution $0.5 \mathrm{~Pa}$ ); and the drag force, by a strain-gage balance (resolution $0.1 \mathrm{~N}$ ). Ambient temperature (wet and dry bulb thermometer, resolution $0.1^{\circ} \mathrm{C}$ ) and atmospheric pressure (digital pressure indicator, accuracy $\pm 500 \mathrm{~Pa}$ ) have also been measured.

The drag coefficient of the model is $C_{\mathrm{DM}}=\frac{T}{1 / 2 \rho V^{2} S_{P}}$, where the reference area is the total area of the two blades.

The Reynolds number is $R e_{P}=V_{v} c / v$. For the tests, it varies between 65,000 and 300,000 approximately.

The velocity ratio is $k=V_{v} /(\omega R)$, where $R=0.138 \mathrm{~m}$.

Flow visualizations have also been performed by means of a stroboscopic lamp and cotton threads attached to the blades.

\section{Configurations Tested}

First, the configuration named "basic configuration" has been tested for determining its behavior. Then, some modifications have been introduced (different blade configurations), and the model has been tested to study the influence of the parameters involved. The aim of testing different blade configurations is to find a way of both reducing the rotation velocity and increasing the drag force over the model, to avoid an excessive rotation velocity over the body that transports the sensors and to slow down its falling velocity as much as possible, respectively.

The so-called basic configuration, CO1, is the one taken as reference, and, particularly, the reference condition is established at a blade pitch angle $\beta=4 \mathrm{deg}$. For this configuration, the blades are bare aluminum alloy rectangular sheets. For the other tested configurations, different elements have been attached to the blades to increase their roughness (rods, sandpaper, etc.), as indicated in Table 1, where the blade pitch settings are also presented.

In the configurations with rods, one or two $5 \mathrm{~mm}$ diameter rods are attached to the surface of the blades in different positions, as can be seen in Fig. 7.
Table 1 Configurations tested

\begin{tabular}{|c|c|c|}
\hline Configuration & Code & Blade pitch angle, deg \\
\hline Basic & $C 01$ & $2,4,6$, and 8 \\
\hline Basic; yaw angle of 10 and $20 \mathrm{deg}$ & $C 01$ & 4 \\
\hline $\begin{array}{l}\text { One rod attached to the lower surface } \\
\text { of the blades }\end{array}$ & $\mathrm{CO} 2$ & 4 and 6 \\
\hline Cylinder without blades & 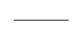 & . \\
\hline Basic; model not allowed to spin & & \\
\hline $\begin{array}{l}\text { Two rods attached to the lower surface } \\
\text { of the blades }\end{array}$ & $\mathrm{CO3}$ & 6 and 8 \\
\hline Rounded leading edge & C04 & 4 \\
\hline Blades with plates attached to the borders & $C 05$ & 2,4 , and 6 \\
\hline Greater diameter & $C 06$ & 2 \\
\hline Increased roughness over the lower surface & $C 07$ & $2,4,6$, and 8 \\
\hline $\begin{array}{l}\text { Two rods attached to the lower surface } \\
\text { of the blades }\end{array}$ & $\mathrm{CO8}$ & 2,4 , and 6 \\
\hline $\begin{array}{l}\text { Two rods attached to the lower surface } \\
\text { of the blades }\end{array}$ & $C 09$ & 4,6 , and 8 \\
\hline
\end{tabular}

The main ideas driving the differences between the characteristics of the configurations tested are the following:

1) The purpose of rounding the leading edge was to avoid the flow detachment at that point.

2) The purpose of the rods was to increase roughness thereby decreasing the rotation velocity of the model.

3) The model without blades was tested for measuring its drag, to be used for theoretical analyses.

4) The purpose of the test with the model fixed (not allowed to spin) was the determination of the drag at that condition.

The configuration $C 07$ has an 80 grains $/ \mathrm{cm}^{2}$ sandpaper attached to the lower surface of the blades.

Concerning the configurations $C 03, C 08$, and $C 09$, all of them have two rods attached to each blade lower surface but placed in different positions (Fig. 7). The first one, C03, has a rod attached close to the leading edge and another at the center of the blades aligned to the span direction. The C08 configuration has one rod attached to the inner edge of the blades and the other one in the central part perpendicular to the flow direction. The $C 09$ configuration has a rod attached at the center of the blades aligned to the span direction and the other one close to the trailing edge of the blades.

\section{Results and Discussion}

The parameters selected for the analysis of the results are the drag coefficient of the model $C_{D M}$ and the velocity ratio $k$.

The results are analyzed as follows: First, the influence of the flow velocity and the blade pitch angle on the parameters $C_{\mathrm{DM}}$ and $k$ is analyzed. Then, the performance of the different configurations is compared with the basic configuration.

As the falling velocity of the device will usually be larger than $8 \mathrm{~m} / \mathrm{s}$ and, generally, both $C_{\mathrm{DM}}$ and $k$ show a constant asymptotic behavior at high speed (see Figs. 8 and 9), the values considered for the analysis are the ones obtained at the highest velocities.

The experimental results obtained are presented in Table 2. Some experiments were repeated several times in similar conditions to verify reproducibility.

\section{Measurement Uncertainty}

Before the presentation of the analysis of the results, the measurement uncertainties analysis is summarized. Measurement uncertainties have been evaluated taking into account the reproducibility of the tests performed in similar conditions and the uncertainties of the instruments. In Table 3 the absolute values of the combined uncertainties (combination of standard deviations) in the determination of $C_{\mathrm{DM}}$ and $k, U_{C_{\mathrm{DM}}}$ and $U_{k}$, respectively, for the basic configuration are presented. For the other tested configurations the combined uncertainties are of about the same order as for the basic configuration.

As it can be seen, $U_{C_{\mathrm{DM}}}>U_{k}$, that is mainly because of variations in the balance calibration. 


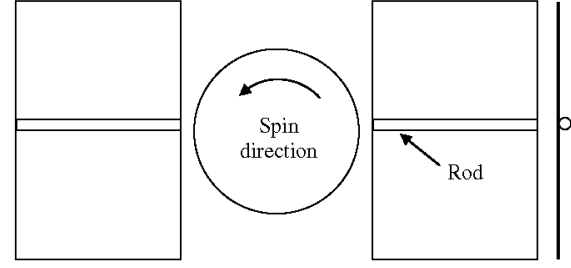

a) $\mathrm{CO2}$

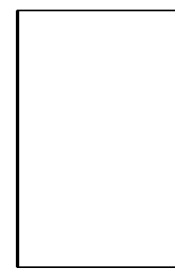

c) $C 05$

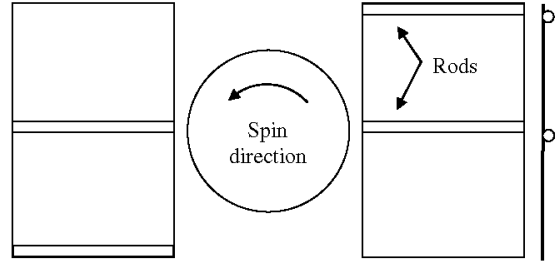

b) $\mathrm{C03}$

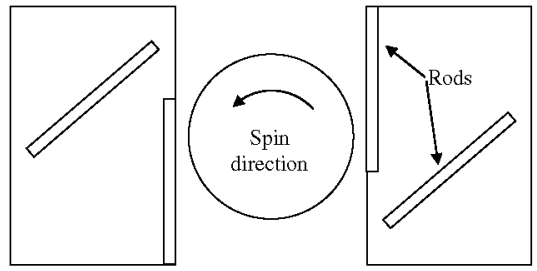

d) $\mathrm{C08}$

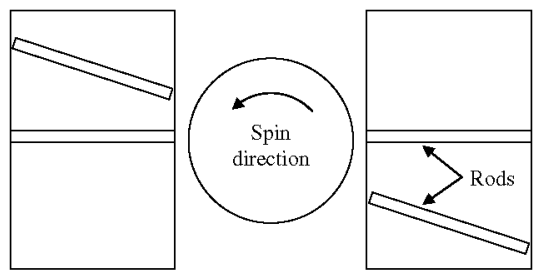

e) $C 09$

Fig. 7 Sketches of configurations.

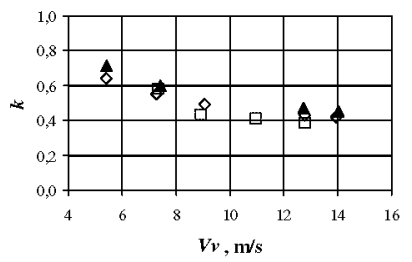

a) $\beta=2 \mathrm{deg}$

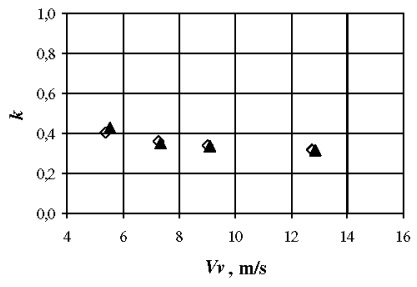

c) $\beta=6 \mathrm{deg}$

Fig. $8 k$ vs $V_{v}$ for the basic configuration. The symbols correspond to different tests performed.

\section{Analysis of the Basic Configuration}

From the values of $C_{\mathrm{DM}}$ and $k$ obtained in the experiments it is observed that 1) $k$ decreases (the rotation velocity increases) when the blade pitch angle and/or the flow velocity increase and 2) $C_{\mathrm{DM}}$ shows small variations with the flow velocity and the blade pitch angle, except for $V_{v}=5.4 \mathrm{~m} / \mathrm{s}$ and $\beta=8 \mathrm{deg}$ where $C_{\mathrm{DM}}$ falls abruptly. For $\beta=8 \mathrm{deg}, C_{\mathrm{DM}}$ values are lower values than for the other blade pitch angles tested.

The results for the basic configuration are shown in Figs. 8 and 9. The complete results can be found in [11].

\section{Analysis of the Other Configurations}

The differences in the parameters $k$ and $C_{\mathrm{DM}}$ obtained for each configuration tested are compared with the values of the reference (basic configuration) and are shown in Table 4 . It is observed that
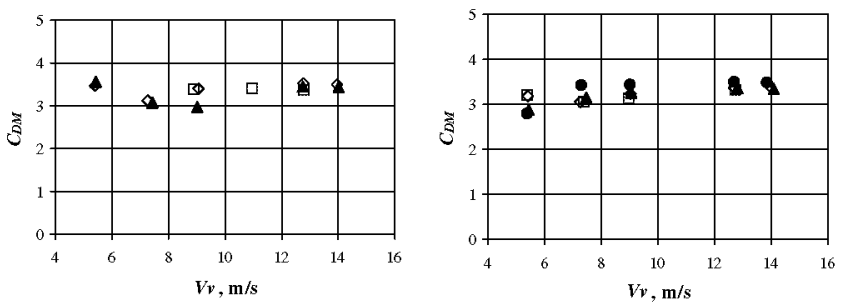

a) $\beta=2 \mathrm{deg}$

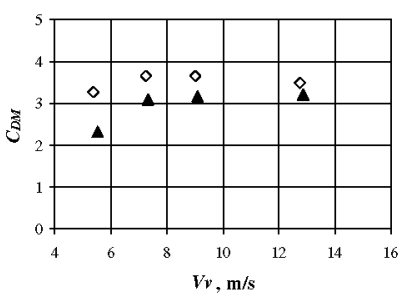

b) $\beta=4$ deg

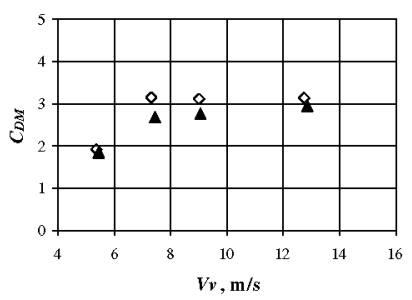

d) $\beta=8 \mathrm{deg}$

c) $\beta=6$ deg

Fig. $9 C_{\mathrm{DM}}$ vs $V_{v}$ for the basic configuration. The symbols correspond to different tests performed.

1) In general, roughness variations have a bigger effect on the parameter $k$ than on $C_{\mathrm{DM}}$ (the rotation velocity falls and the drag coefficient of the model is nearly unchanged).

2) As the yaw angle increases, $k$ increases and $C_{\mathrm{DM}}$ decreases.

3 ) Rounding the leading edge produces no changes ( $\mathrm{CO4}$ ).

4) A larger diameter of the device results in an increase in $C_{\mathrm{DM}}$, although this configuration could not be fully explored.

5) A higher roughness in the lower surface, generated by means of sandpaper attached to the blades $(\mathrm{C} 07)$, produces a small increase in $C_{\mathrm{DM}}$, when the blade pitch angle is at both sides ( $\beta=2$ and $8 \mathrm{deg}$ ) of the tested range.

6) The configuration with two rods attached to the lower surface (C09) shows the largest increase in the parameter $k$ (125\% for $\beta=4 \mathrm{deg}) . C_{\mathrm{DM}}$ decreases $7 \%$.

7) The other tested configurations ( $\mathrm{CO2}, \mathrm{CO3}, \mathrm{CO5}$, and $\mathrm{C08}$ ) show an increase in $k$ between 21 and $87 \%$ and a small change in $C_{\mathrm{DM}}$. 
Table 2 Asymptotic values for $k$ and $C_{\mathrm{DM}}$

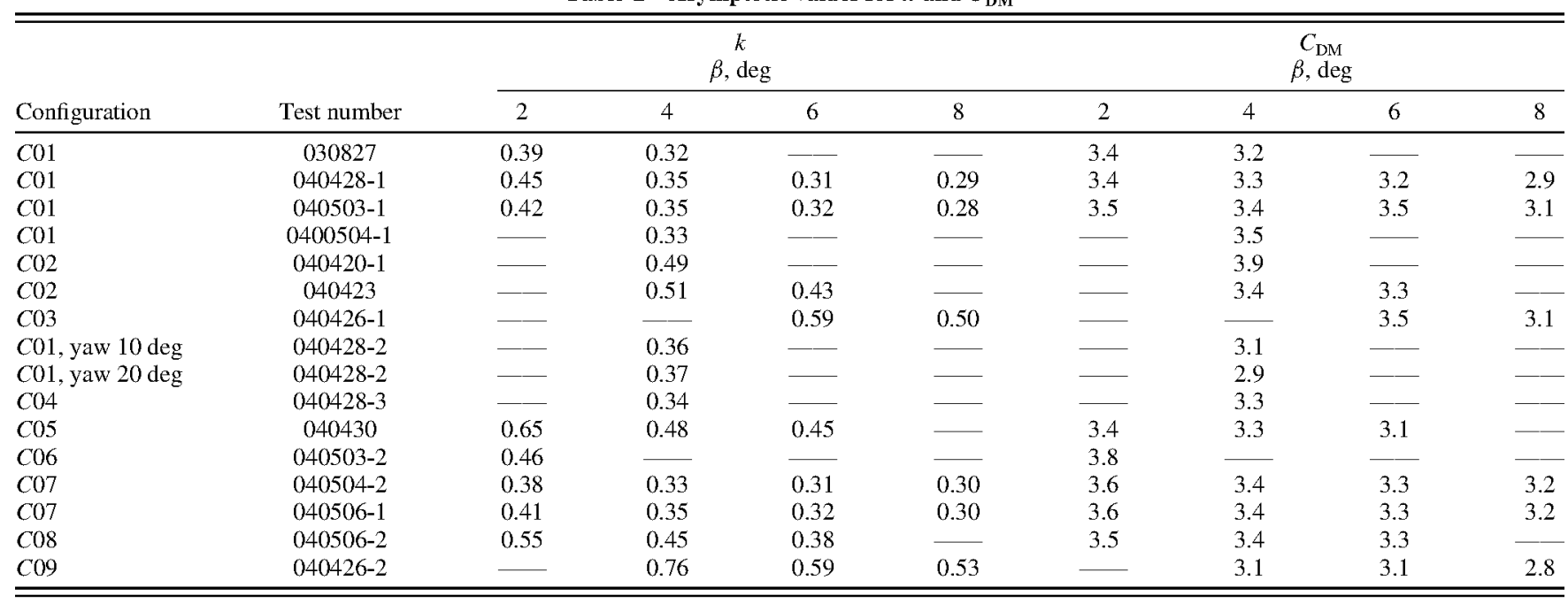

Table 3 Measurement combined uncertainty for the basic configuration

\begin{tabular}{|c|c|c|c|c|c|c|c|c|}
\hline \multirow[b]{2}{*}{$V_{v}, \mathrm{~m} / \mathrm{s}$} & \multicolumn{4}{|c|}{$\begin{array}{c}U_{C_{\mathrm{DM}}} \\
\beta, \operatorname{deg}\end{array}$} & \multicolumn{4}{|c|}{$\begin{array}{c}U_{k} \\
\beta, \operatorname{deg}\end{array}$} \\
\hline & 2 & 4 & 6 & 8 & 2 & 4 & 6 & 8 \\
\hline 7.3 & 0.14 & 0.16 & 0.33 & 0.24 & 0.018 & 0.008 & 0.008 & 0.005 \\
\hline 9.0 & 0.17 & 0.13 & 0.27 & 0.18 & 0.031 & 0.008 & 0.006 & 0.005 \\
\hline 12.8 & 0.08 & 0.09 & 0.17 & 0.13 & 0.025 & 0.009 & 0.005 & 0.005 \\
\hline 14.0 & 0.09 & 0.10 & - & - - & 0.017 & 0.008 & - & - \\
\hline
\end{tabular}

Table 4 Variations of the parameters $k$ and $C_{\mathrm{DM}}$ with regard to the basic configuration

\begin{tabular}{|c|c|c|c|c|c|c|c|c|}
\hline \multirow[b]{2}{*}{ Configuration } & \multicolumn{4}{|c|}{$\begin{array}{c}\text { Variation of } k, \% \\
\beta, \operatorname{deg}\end{array}$} & \multicolumn{4}{|c|}{$\begin{array}{c}\text { Variation of } C_{\mathrm{DM}}, \% \\
\beta, \text { deg }\end{array}$} \\
\hline & 2 & 4 & 6 & 8 & 2 & 4 & 6 & 8 \\
\hline $\mathrm{CO3}$ & - & - & 87 & 75 & - & - & 4 & 3 \\
\hline C01, yaw 10 deg & - & 7 & - & - & - & -7 & - & —— \\
\hline C01, yaw 20 deg & - & 10 & - & - & - & -13 & - & - - \\
\hline $\mathrm{CO} 4$ & - & 1 & - & - & - & -1 & - & - \\
\hline$C 07$ & -6 & 1 & 0 & 5 & 5 & 1 & -1 & 7 \\
\hline C08 & 31 & 33 & 21 & - & 2 & 1 & -1 & - \\
\hline$C 09$ & - & 125 & 87 & 86 & - & -7 & -7 & -7 \\
\hline
\end{tabular}

\section{E. Flow Visualizations}

The flow pattern is almost steady when it is seen in body axes. It is difficult to show this phenomenon because of the complexity to attach a visualization system to the body axes, and so a device that registers in phase with the rotation of the body has been used. The aim is to observe whether the flow is attached to the surface of the blades or not and to observe the flow direction over the blades. To do so, cotton threads have been attached to the blades and illuminated by using a stroboscopic lamp. It is observed that, for every configuration, the overall flow pattern does not change, neither with the blade pitch angle nor with the flow velocity. Generally speaking, the pattern observed is a flow attached to the upper and lower surfaces of the blades, except along the leading edge at the upper surface, where a vortex that grows outwards along the span is observed. At the inner portion of the lower surface of the leading edge, an exiguous flow towards the upper surface is observed. At the leading edge, the flow has a prevailing radial direction; at the trailing edge, it follows the chord direction; and in the central part of the blades, the flow is at $45 \mathrm{deg}$ from the radial direction. Figure 10 shows a photograph taken of the model. The numbers in the figure indicate the following: 1 is the spin direction; 2 is the flow direction; and 3 is the threads that indicate the flow direction over the lower surface of the blade. Because of the difficulty of obtaining good quality pictures, the general flow pattern is reproduced schematically in Figs. 11 and 12.

It has been observed that

1) Rounding the leading edge does not avoid the flow detachment at that place.

2) The rods produce small perturbations of the flow only in their surroundings (Fig. 12). When placed at the leading edge on the lower surface, they do not avoid flow detachment there.

For configurations $\mathrm{C06}, \mathrm{C07}$, and $\mathrm{C} 08$, the flow was not visualized.

\section{F. Conclusions on Experimental Results}

As mentioned, for the basic configuration, the velocity ratio $k$ decreases (the rotation velocity increases) when the blade pitch angle and/or the flow velocity increase; and $C_{\mathrm{DM}}$ shows a small variation with the flow velocity and the blade pitch angle, except for 


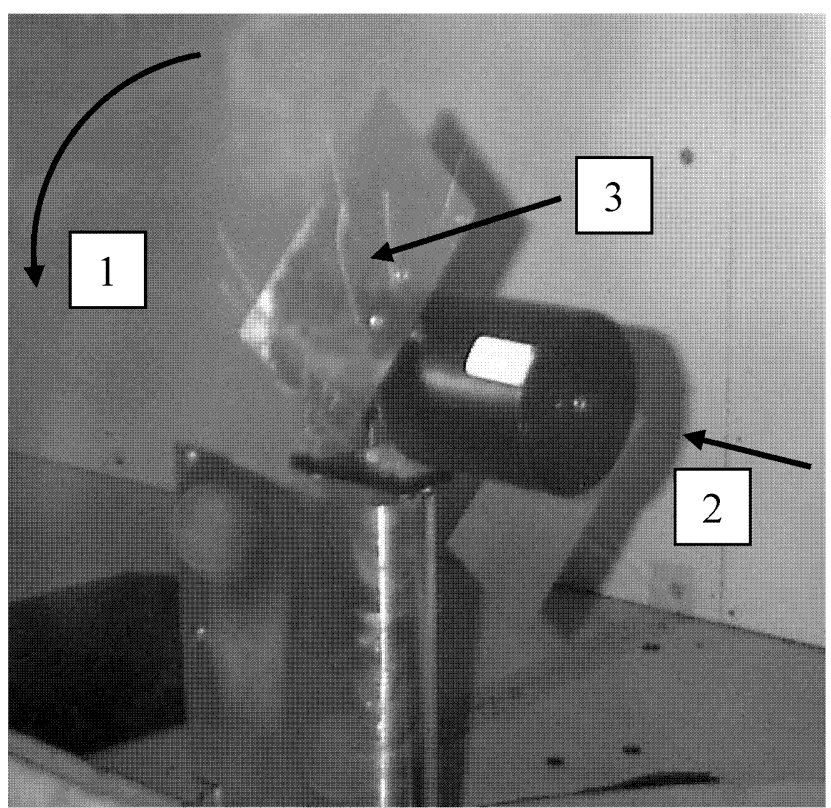

Fig. 10 Flow visualization at the lower surface for the basic configuration.

Flow towards the

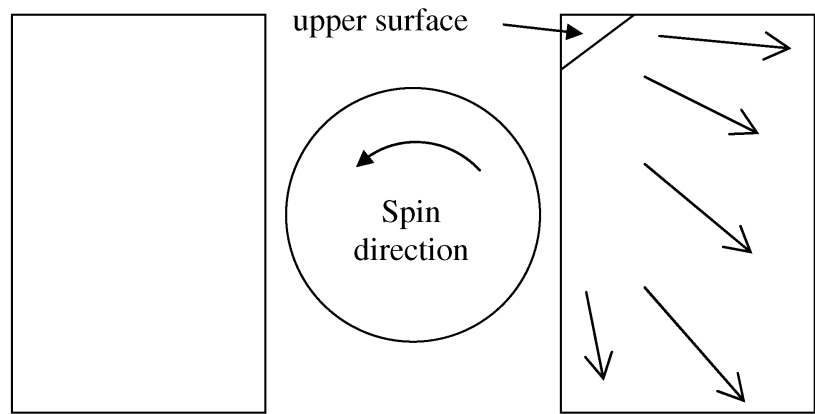

a) Lower surface, view from upstream site

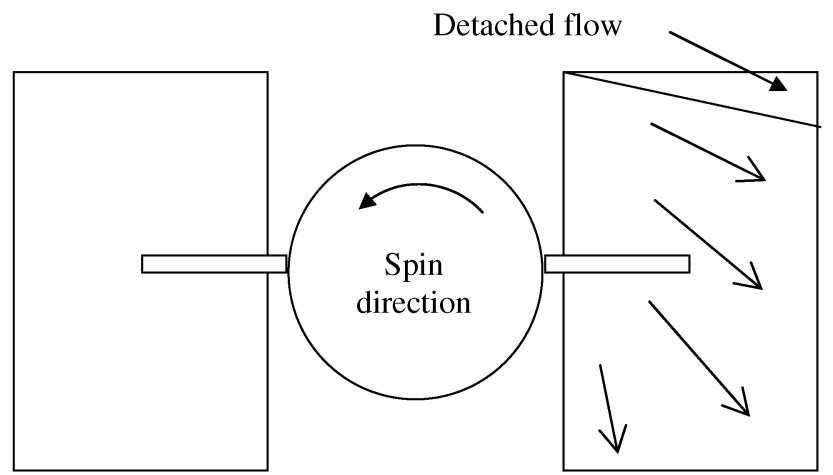

b) Upper surface, view from downstream site

Fig. 11 Sketch of the flow on the surfaces for the C01 configuration.

$V_{v}=5.4 \mathrm{~m} / \mathrm{s}$ and $\beta=8 \mathrm{deg}$ where $C_{\mathrm{DM}}$ falls abruptly. For $\beta=8 \mathrm{deg}, C_{\mathrm{DM}}$ gives lower values than for the other blade pitch angles tested.

When the other configurations are compared with the basic configuration, it is observed that the velocity ratio increases for every configuration except for some cases of the $C 07$ configuration (sandpaper attached to the lower surface).

The drag coefficient of the model does not show a well defined behavior. Generally speaking, this parameter decreases, except for some cases: the $C 03$ configuration (two rods attached to the lower surface); the C06 configuration (greater diameter), although this

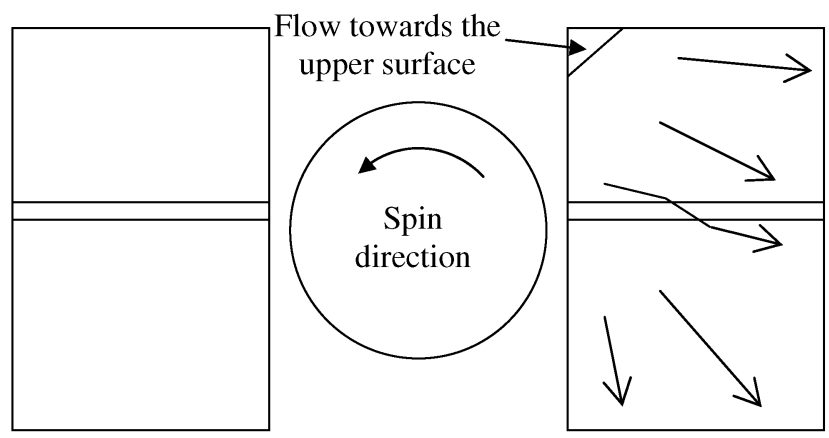

a) Lower surface, view from upstream site

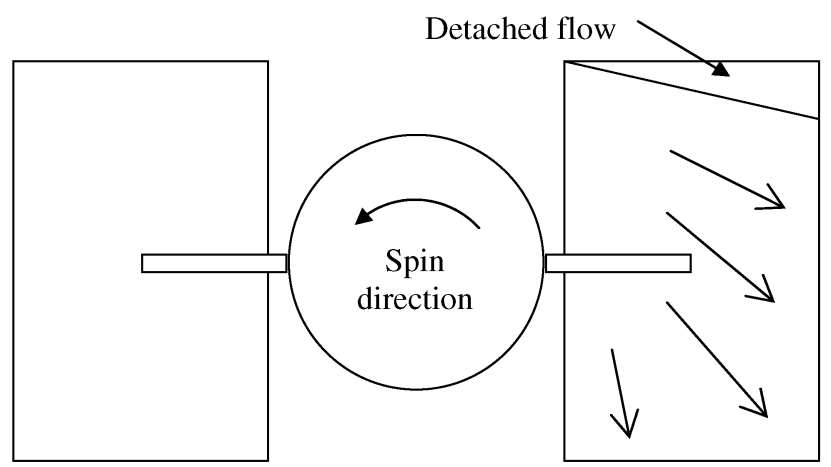

b) Upper surface, view from downstream site

Fig. 12 Sketch of the flow on surfaces for the $\mathrm{C02}$ configuration.

configuration was not fully explored; and the $C 07$ configuration (sandpaper attached to the lower surface). The maximum changes for the drag coefficient of the model are about $10 \%$ and for the velocity ratio up to $125 \%$. The reproducibility of the tests is considered appropriate.

Concerning the visualization of the flow, the flow patterns change neither with the blade pitch angle nor with the flow velocity, and they also do not change significantly for the different configurations tested (the rods only produce small changes in the direction of the flow locally, very close to them). The flow is attached along almost all the surface of the upper and lower faces of the blades. Furthermore, the flow is steady, considered in the body reference frame.

Finally, it is concluded that if it is desired to increase the velocity ratio, that is, to decrease the rotation velocity, the roughness of the blades should be increased, as predicted by the theoretical model. In the same way, small values of the blade pitch angle are appropriate to obtain this effect, which is also good to produce an increase of the drag coefficient of the model $C_{\mathrm{DM}}$.

\section{Determination of the Free Parameters of the Theoretical Model}

As mentioned, the approach followed to determine the free parameters of the theoretical model is to find the values of the coefficients $C_{D 0}, a$, and $C_{L \alpha}$ that give the best agreement between both the experimental results and the predictions of the model. The approach followed consists of these steps: processing of the experimental data, selection of the data, and matching of the results.

\section{A. Processing of the Experimental Data}

To carry out the matching in a proper way, the experimental data is processed as follows:

1) The theoretical model does not take into account the drag of the cylinder, which has been measured and subtracted from the measured drag of the whole body.

2) The induced velocity at hover is calculated by

$$
v_{i o}=\sqrt{\frac{T}{2 \rho S_{D}}}
$$


3) The tangential velocity is taken at $60 \%$ of the blades span $(0.75 R)$. It is assumed that the center of pressure is located at that position. This assumption comes from analyzing the flow visualizations, the literature [11], and the results of the matching.

\section{B. Selection of the Data}

The data used for determining the values of the parameters of the theoretical model that give rise to a better fit among the model predictions and the experimental results come from the tests performed with the configurations $C 01, C 03, C 07$, and $C 09$. These configurations have been tested several times and correspond to the extreme values of roughness. The data considered correspond to the highest flow velocity tested (asymptotic value). The processed experimental data used for the matching are shown in Tables 5-8. The values of $k$ and $\overline{U_{T}^{2}}$ are obtained from the experimental data considering $r=R$.

\section{Matching of the Theoretical Model and Experimental Results}

The values of $C_{D 0}, a$, and $C_{L \alpha}$ that give the smaller differences between the theoretical and experimental parameters ${\overline{U_{T}}}^{2}, k$, and $-\overline{V_{v}}$ are found by using a least-mean-square method. To do so, first a "normalized distance" $B$ is defined as follows:

$$
B=\frac{\sum_{i=1}^{3} \operatorname{dif}\left(p_{i}\right)}{3}
$$

Table 5 Experimental data; configuration $C 01, r=R$

\begin{tabular}{lccc}
\hline \hline$\beta, \operatorname{deg}$ & $k$ & $\overline{V_{v}}$ & $\overline{U_{T}^{2}}$ \\
\hline 2 & 0.42 & 1.74 & 16 \\
4 & 0.34 & 1.76 & 26 \\
6 & 0.32 & 1.77 & 31 \\
8 & 0.29 & 1.86 & 42 \\
\hline \hline
\end{tabular}

Table 6 Experimental data; configuration $C 03, r=R$

\begin{tabular}{lccc}
\hline \hline$\beta, \operatorname{deg}$ & $k$ & $\overline{V_{v}}$ & $\overline{U_{T}^{2}}$ \\
\hline 6 & 0.61 & 1.74 & 9 \\
8 & 0.52 & 1.85 & 14 \\
\hline \hline
\end{tabular}

Table 7 Experimental data; configuration $C 07, r=R$

\begin{tabular}{lccc}
\hline \hline$\beta, \operatorname{deg}$ & $k$ & $\overline{V_{v}}$ & $\overline{U_{T}^{2}}$ \\
\hline 2 & 0.41 & 1.72 & 19 \\
4 & 0.36 & 1.70 & 27 \\
6 & 0.33 & 1.80 & 33 \\
8 & 0.31 & 1.82 & 37 \\
\hline \hline
\end{tabular}

Table 8 Experimental data; configuration $C 09, r=R$

\begin{tabular}{lccc}
\hline \hline$\beta, \operatorname{deg}$ & $k$ & $\overline{V_{v}}$ & $\overline{U_{T}^{2}}$ \\
\hline 4 & 0.79 & 1.85 & 6 \\
6 & 0.62 & 1.86 & 10 \\
8 & 0.56 & 1.96 & 14 \\
\hline \hline
\end{tabular}

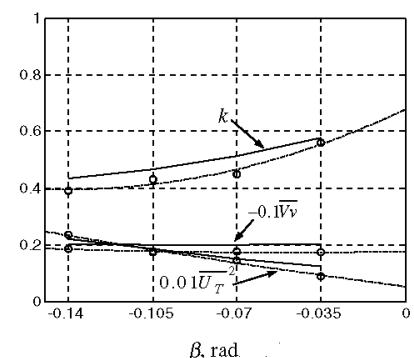

a) Configuration $C 01$

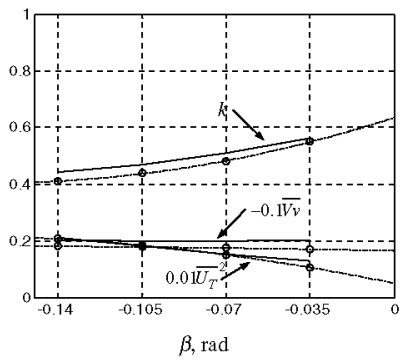

c) Configuration $\mathrm{C} 07$

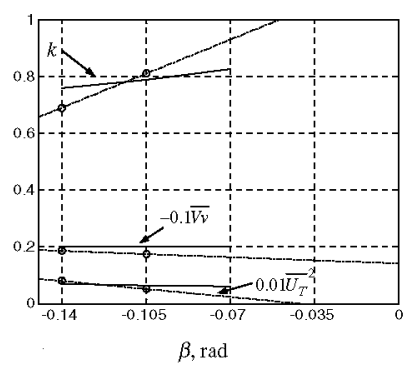

b) Configuration $\mathrm{CO3}$

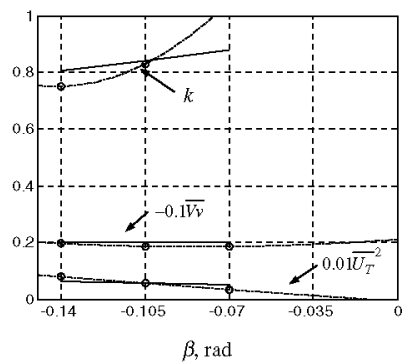

d) Configuration $\mathrm{C} 09$
Fig. 13 Matching of theoretical model predictions (solid lines) and experimental data (circles). Dashed-dotted lines show a fitting curve to the experimental data.

where $\operatorname{dif}\left(p_{i}\right)$ is the relative mean difference between the theoretical and experimental values for each parameter $p_{i}$, i.e., $p_{1}=k$, $p_{2}=-\overline{V_{v}}, p_{3}={\overline{U_{T}}}^{2}$, and it is given by

$$
\operatorname{dif}\left(p_{i}\right)[\%]=100 \frac{\sqrt{\sum_{j=1}^{N}\left[p_{i \exp }\left(\beta_{j}\right)-p_{i t h e}\left(\beta_{j}\right)\right]^{2}} / N}{\sum_{j=1}^{N} p_{i \exp }\left(\beta_{j}\right) / N}
$$

where $p_{i \exp }\left(\beta_{j}\right)$ is the experimental value for the parameter $p_{i}$ for the blade pitch angle $\beta_{j}, p_{i \text { the }}\left(\beta_{j}\right)$ is the theoretical value for the parameter $p_{i}$ for the blade pitch angle $\beta_{j}$, and $N$ is the number of cases considered for each $p_{i}$ (it is the number of blade pitch angles tested for each configuration).

As mentioned, the coefficients $C_{D 0}, a$, and $C_{L \alpha}$ found in this way are the ones that minimize the normalized distance $B$ and, therefore, they give rise to the best agreement between the theoretical model and the experimental results. These results are shown in Table 9 and Fig. 13.

\section{Conclusions on the Matching of the Theoretical Model and the Experiments}

It is observed that

1) Increasing the blade roughness leads to an increase on $C_{D 0}$ ranging from 0.07 and 0.08 for the lower roughness configurations ( $C 01$ and $C 07$, respectively) to 0.35 and 0.38 for the higher ones (C03 and C09, respectively).

2) The parameter $a$ varies between 2 and 2.7. It is about the magnitude of the corresponding ones found in [12] for a rectangular flat plate of aspect ratio 3 .

3) The relative mean differences between the theoretical values and the experimental results of the parameters $k,-\overline{V_{v}}$, and ${\overline{U_{T}}}^{2}$ lie under $10 \%$ for most of the cases analyzed. A better agreement had not been possible to attain, probably because of both the simplifying assumptions considered to develop the theoretical model and the

Table 9 Matching of the theoretical model and the experimental results

\begin{tabular}{lcccccccc}
\hline \hline Configuration & $C_{D 0}$ & $a$ & $C_{L \alpha}$ & $\operatorname{dif}(k), \%$ & $\operatorname{dif}\left(\overline{V_{\nu}}\right), \%$ & $\operatorname{dif}\left({\overline{U_{T}}}^{2}\right), \%$ & $B, \%$ & $N$ \\
\hline C01 & 0.07 & 2.4 & 3.4 & 4.7 & 6.6 & 6.0 & 5.7 & 4 \\
$C 03$ & 0.35 & 2.3 & 4.8 & 4.7 & 8.4 & 12.3 & 8.4 & 2 \\
$C 07$ & 0.08 & 2.0 & 3.4 & 3.0 & 7.0 & 4.0 & 4.6 & 4 \\
$C 09$ & 0.38 & 2.7 & 4.6 & 7.0 & 3.7 & 14.5 & 8.4 & 3 \\
\hline \hline
\end{tabular}


experiment uncertainties. However, the result of the matching is considered good enough for the purpose.

It should be outlined that the better agreement occurs for the low drag cases ( $\mathrm{CO} 1$ and $\mathrm{C} 07$ ), whereas it seems to be poorer for the large drag cases ( $\mathrm{CO3}$ and $\mathrm{C} 09$ ). This difference could be due to the large values of the angle of attack that appear in configurations with large drag (Fig. 5a), values that are close to the limit of validity of the simple model employed to describe the $C_{L}$ vs $\alpha$ relation.

\section{Conclusions}

Research concerning the aerodynamic behavior of a pararotor has been carried out leading to the development of a semi-empirical model. The theoretical part of the model allows us to determine both the falling and rotation velocities and also the drag generated on a pararotor that falls in the atmosphere. The input data needed are three parameters: the blade drag coefficient, the slope of the lift vs angleof-attack curve for the blades, and the rotor solidity. The model describes the behavior of the rotation and falling velocities and the drag of the pararotor as a function of the blade pitch angle, the solidity ratio, and the aerodynamic characteristics of the blades. The model can also be used for evaluating the influence of the input parameters on the performance of the device.

On the other hand, a set of experimental tests has been carried out to support the development of the theoretical model (results of tests similar to these could not be found in the literature). One type of model has been tested, maintaining the same planform but with different roughness. These tests give information about the experimental performance of the device, in particular, about the effect of the blade roughness, the blade pitch angle, and the flow velocity on both the rotation velocity and the drag of the model. The agreement between the theoretical model and the experimental data is satisfactory for reasonable values of the aerodynamic coefficients, which are obtained from the matching.

Flow visualizations have allowed us to identify the characteristics of the flow pattern around the blades and to make more precise hypotheses for the development of the theoretical model as well.

In this way, this work also contributes to the knowledge of the behavior of low aspect ratio rotating-winged devices, which could be

useful for several applications, as decelerating devices for atmospheric probes, final phase of small reentry vehicles, submunition, etc.

\section{References}

[1] Crimi, P., "Analysis of Samara-Wing Decelerator Steady-State Characteristics," Joumal of Aircraft, Vol. 25, No. 1, 1988, pp. 41- 47.

[2] Karlsen, L., Borgström, D., and Paulsson, L., "Aerodynamics of a Rotating Body Descending from the Separation Position of an Artillery Munition Shell," AIAA Paper 91-0870-CP, April 1991.

[3] Pepper, W., Jr., "A New Rotating Parachute Design Having High Performance," Journal of Spacecraft and Rockets, Vol. 23, No. 2, 1986, pp. 222-224.

[4] Shpund, Z., and Levin, D., "Measurement of the Static and Dynamic Coefficients of a Cross-Type Parachute in Subsonic Flow," AIAA Paper 91-0871-CP, April 1991.

[5] Levin, D., and Shpund, Z., "Dynamic Investigation of the Angular Motion of a Rotating Body-Parachute System," Joumal of Aircraft, Vol. 32, No. 1, 1995, pp. 93-99.

[6] Shpund, Z., and Levin, D., "Forebody Influence on Rotating Parachute Aerodynamic Properties," Journal of Aircraft, Vol. 34, No. 2, 1997 , pp. 181-186.

[7] Levin, D., and Shpund, Z., "Canopy Geometry Effect on the Aerodynamic Behavior of Cross-Type Parachutes,"Journal of Aircraft, Vol. 34, No. 5, 1997, pp. 648-652.

[8] Rosen, A., and Seter, D., "Vertical Autorotation of a Single Winged Samara," Journal of Applied Mechanics, Vol. 58, No. 4, Dec. 1991, pp. 1064-1070

[9] Seter, D., and Rosen, A., "Stability of the Vertical Autorotation of a Single Winged Samara," Journal of Applied Mechanics, Vol. 59, No. 4, Dec. 1992, pp. 1000-1008.

[10] Stahl, W. H., "Aerodynamics of Low Aspect Ratio Wings," Missile Aerodynamics, Vol. 98, AGARD Lecture Series, AGARD, Paris, 1979, pp. 1-64.

[11] Nadal Mora, V., "Comportamiento Aerodinámico de Sondas Atmosféricas en Entornos Aeroportuarios," Doctoral Tesis, Anexo I, Universidad Politécnica de Madrid, Madrid, Spain, 2005, pp. Anexo 1.2-Anexo 1.19 .

[12] Pelletier, A., and Mueller, T. J., "Low Reynolds Number Aerodynamics of Low-Aspect-Ratio, Thin/Flat/Cambered-Plate Wings," Journal of Aircraft, Vol. 37, No. 5, 2000, pp. 825-832. 